\title{
COVID-19 vaccination rates and SARS-CoV-2 infection in pregnant women in Scotland
}

Sarah Stock ( $\square$ sarah.stock@ed.ac.uk)

University of Edinburgh https://orcid.org/0000-0003-4308-856X

Jade Carruthers

Public Health Scotland

Clara Calvert

University of Edinburgh

Cheryl Denny

Public Health Scotland

Jack Donaghy

Public Health Scotland

Anna Goulding

Public Health Scotland

Lisa Hopcroft

Public Health Scotland

Leeanne Hopkins

Public Health Scotland

Terry McLaughlin

Public Health Scotland

Jiafeng Pan

Public Health Scotland

\section{Ting Shi}

Usher Institute, University of Edinburgh https://orcid.org/0000-0002-4101-4535

John Taylor

Public Health Scotland https://orcid.org/0000-0003-4314-2685

Utkarsh Agrawal

School of Medicine, University of St Andrews

\section{Bonnie Auyeung}

University of Edinburgh

Srinivasa Katikireddi

University of Glasgow https://orcid.org/0000-0001-6593-9092

Colin McCowan

School of Medicine, University of St Andrews

Josie Murray 
Public Health Scotland

\section{Colin Simpson}

Victoria University of Wellington https://orcid.org/0000-0002-5194-8083

\section{Chris Robertson}

Public Health Scotland

\section{Eleftheria Vasileiou}

University of Edinburgh

\section{Aziz Sheikh}

University of Edinburgh https://orcid.org/0000-0001-7022-3056

\section{Rachael Wood}

Usher Institute, University of Edinburgh

\section{Brief Communication}

Keywords: SARS-CoV-2, COVID-19 vaccine uptake, pregnancy

Posted Date: November 9th, 2021

DOl: https://doi.org/10.21203/rs.3.rs-1051010/v1

License: (c) (7) This work is licensed under a Creative Commons Attribution 4.0 International License. Read Full License

Version of Record: A version of this preprint was published at Nature Medicine on January 13th, 2022. See the published version at https://doi.org/10.1038/s41591-021-01666-2. 


\section{Abstract}

We describe SARS-CoV-2 infection and COVID-19 vaccine uptake in Scotland in a prospective cohort of all pregnant women in Scotland drawn from national databases. As of mid-October 2021, the Covid-19 in pregnancy in Scotland (COPS) cohort included linked data on a total of 139,136 pregnancies in 126,749 women. Up to September 30, 2021, a total of 22,779 COVID-19 vaccinations had been administered to 16,229 pregnant women. Vaccine coverage was substantially lower in pregnant women than in the general female population of reproductive age (23.7\% of women giving birth in September 2021 were fully vaccinated compared to $74.9 \%$ in women $18-44$ years). Of the 4,274 cases of COVID-19 in pregnancy (confirmed by SARS-CoV-2 viral reverse transcriptase polymerase chain reaction) between December 2020 (the month the COVID-19 vaccination programme started in Scotland) and September 2021 inclusive, 629 women (14.7\%) were admitted to hospital and $89(2.1 \%)$ were admitted to critical care. Of the COVID-19 cases occurring in pregnant women, 81.7\% (3,491/4,274; 95\% Cl 80.5-82.8) were in unvaccinated women. Of the COVID-19 associated hospital admissions, 93.0\% (585/629; 95\% Cl 90.794.8) were in women who were unvaccinated at the time of COVID-19 diagnosis. Of the COVID-19 associated critical care admissions $98.9 \%(88 / 89 ; 95 \% \mathrm{Cl}$ 93.9-100) were in women who were unvaccinated at the time of COVID-19 diagnosis. The extended perinatal mortality rate for women who gave birth within 28 days of COVID-19 diagnosis was 15.9 per 1000 births ( $95 \% \mathrm{Cl} 7.8$ to 31.0 ; background rate in 20206.3 per 1,000 total births [95\% Cl 5.7-7.1]; background rate 20195.7 per 1,000 total births [95\% $\mathrm{Cl} 5.0-6.4]$ ). All baby deaths occurred after pregnancies in women who were unvaccinated at the time of COVID-19 diagnosis. Addressing low vaccine uptake rates in pregnant women is imperative to protect the health of women and babies.

\section{Main Article}

Pregnant women are at higher risk of severe COVID-19 disease than non-pregnant women ${ }^{1}$ and COVID-19 in pregnancy is associated with increased risk of pre-eclampsia, preterm birth and stillbirth. ${ }^{2-8}$ All evidence from studies that include pregnant women indicates that COVID-19 vaccines are safe in pregnancy. ${ }^{9}$ Although vaccine hesitancy in pregnant populations is well recognized, comprehensive whole population data on SARS-CoV-2 infection rates and COVID-19 vaccine uptake in pregnancy are lacking. ${ }^{10}$

The COVID-19 vaccination program in Scotland commenced on December 8, 2020. Policies on the provision of COVID-19 vaccination to pregnant women in Scotland have evolved over time (Table S1); currently, women who are pregnant are offered vaccination at the same time as non-pregnant women, based on their age and clinical risk group.

Here we describe SARS-CoV-2 infection and COVID-19 vaccine uptake in Scotland, using population data from a national, prospective dynamic cohort (the COVID-19 in Pregnancy in Scotland (COPS) study; ${ }^{11,12}$ a sub-study of EAVE II (Early Pandemic Evaluation and Enhanced Surveillance of COVID-19). ${ }^{13,14}$ COPS 
includes all women who were pregnant on, or became pregnant after, March 1, 2020; technical details and study ethical and governance approval details are summarized in Table S2.

In COPS, we have linked national healthcare data from women who had early pregnancy loss, termination of pregnancy, live or stillbirth, neonatal death, or antenatal care for an ongoing pregnancy with national data on COVID-19 vaccination and confirmed SARS-CoV-2 infection (positive viral reverse transcriptase polymerase chain reaction [RT-PCR] test) (details of datasets are in Table S2 and ${ }^{11,12}$ ). As of midOctober 2021, the COPS database included linked data on a total of 139,136 pregnancies in 126,749 women.

\section{COVID-19 rates in pregnancy in Scotland}

Up to September 30, 2021, there were 4,979 cases of SARS-CoV-2 in pregnant women. Throughout the pandemic, rates of confirmed SARS-CoV-2 in pregnancy showed similar patterns to those in the general female population of reproductive age, with peaks of infection in October 2020 and January 2021, and high infection rates in July and August 2021, which continued to increase in pregnant women to the time of latest data release in September 2021 (Figure 1) (numbers of infections presented in Table S3). Infection rates have been consistently highest in pregnant women living in the most deprived areas, and in younger, compared to older, pregnant women (Figure S1), similar to patterns seen in the general population. ${ }^{15}$

The percentage of SARS-CoV-2 infections that were temporally associated with a hospital admission (i.e., admission within 14 days of positive COVID-19 RT-PCR) or critical care admission (i.e. admission within 21 days of positive COVID-19 RT-PCR) was higher in pregnant women than those in the general female population age 18-44 years (Figure 1). This is likely to reflect the fact that admission is generally more common among pregnant than non-pregnant women (due to admissions for obstetric complications, labour and birth; as well as potentially due to a lower threshold for admission for COVID-19 in pregnant women than for COVID-19 in the general population), and also that COVID-19 is more likely to cause severe disease in pregnant than non-pregnant women. ${ }^{1}$

Although surveillance studies (mainly based on women admitted to hospital) have suggested that pregnant women are at highest risk of COVID-19 in the third trimester, ${ }^{8}$ these Scottish population data capturing all virologically confirmed infections in a setting where testing has been widely available in hospital and the community - show SARS-CoV-2 infections were relatively evenly spread throughout pregnancy: $1,592(31.9 \% 95 \% \mathrm{Cl} 30.1-33.3)$ onset in the first trimester of pregnancy [ $2+0$ to $13+6$ weeks gestation]; $1,875(37.6 \% 95 \% \mathrm{Cl} 36.3-39.0)$ in the second trimester [14+0 to $27+6$ weeks gestation]; and 1,512 (30.4\% 95\% Cl 29.1-31.2) in the third trimester [at 28+0 weeks gestation or over]. However, COVID19 associated with hospital and critical care admission was more common as pregnancy progressed, with hospital admission rates of $6.5 \%$ (103/1,592 cases; $95 \%$ Cl 5.3-7.8) in first trimester, $9.1 \%(170 / 1,875$ cases; $95 \% \mathrm{Cl} 7.8-10.5)$ in the second trimester and $30.4 \%(460 / 1,512 ; 95 \% \mathrm{Cl} 28.1-32.8)$ in the third trimester; and critical care admission rates of $0 \%(0 / 1,592$ cases; $95 \% \mathrm{Cl} 0-0.3)$ in the first trimester, $1.8 \%$ 
$(33 / 1,875$ cases; $95 \% \mathrm{Cl} 1.2-2.5)$ in the second trimester and $4.4 \%(66 / 1,512 ; 95 \% \mathrm{Cl} 3.4-5.6)$ in the third trimester.

\section{COVID-19 vaccine coverage and uptake in Scotland}

Up to September 30, 2021, a total of 22,779 primary COVID-19 vaccinations (12,030 [53\%] first doses and 10,749 [47\%] second doses) had been given to 16,229 pregnant women in Scotland: 8,945 (39.3\%; $95 \% \mathrm{Cl}$ 38.6-39.9) vaccinations were given in the first trimester of pregnancy ( $2^{+0}$ to $13^{+6}$ weeks gestation); 8,030 (35.3\%; $95 \% \mathrm{Cl} 34.6-35.9)$ in the second trimester $\left(14^{+0}\right.$ to $27^{+6}$ weeks gestation); and 5,804 (25.5\%; $95 \%$ $\mathrm{Cl} 24.9-26.1)$ in the third trimester ( $28^{+0}$ weeks gestation or over). 17,841 (78.3\%; $\left.95 \% \mathrm{Cl} 77.8-78.9\right)$ of the vaccinations given were Pfizer-BioNTech BNT162b2 mRNA vaccine; 2,853 (12.3\%; 95\% Cl 12.1-13.0) were Moderna mRNA-1273 mRNA vaccine; and 2,085 (9.2\%; 95\% Cl 8.8-9.5\%) were Oxford-AstraZeneca AZD1222 viral vector vaccine.

Coverage of vaccination (defined as the percentage of women who were vaccinated at some point prior to giving birth i.e. vaccination may have been given before or during pregnancy) increased over time, but coverage has been consistently substantially lower than coverage in the same age groups seen in the general female population (Figure 2). 3,992 women gave birth in September 2021, of whom 1,342 (33.6\%; 95\% Cl 32.2-35.1) had received any COVID-19 vaccination prior to birth, with $946(23.7 \%$; $95 \%$ Cl 22.425.1 ) of the women having received two primary doses of vaccination. In contrast, by the end of September 2021, 83.6\% (792,136/947,984; 95\% Cl 83.5-83.63]) of women aged 18-44 years in the general population had received any vaccination, and $74.9 \%$ had received two doses $(710,219 / 947,984 ; 95 \% \mathrm{Cl}$ 74.8-75.0).

Vaccine uptake (i.e. the percentage of pregnant women receiving a vaccine each month) was consistently lower than the corresponding percentage of the general population of women of reproductive age (Figure 2). Vaccine uptake was lowest in younger pregnant women and those living in the most deprived areas of Scotland (Figure S2), similar to patterns seen in the general population. ${ }^{15}$

\section{SARS-CoV-2 infection and severe COVID-19 outcomes in vaccinated and unvaccinated pregnant women}

Of the 4,274 cases of SARS-CoV-2 in pregnancy (as confirmed by positive RT-PCR) between December 2020 (the month the COVID-19 vaccination programme started in Scotland) and September 2021 inclusive, 629 women were admitted to hospital within 14 days of COVID-19 diagnosis (14.7\%; $95 \%$ CI 13.7-15.8) and 89 (2.1\%; 95\% Cl 1.7-2.6) were admitted to critical care within 21 days of diagnosis. 81.7\% $(3,491 / 4,274 ; 95 \%$ Cl 80.5-82.8) of COVID-19 cases, 93.0\% (585/629; 95\% Cl 90.7-94.9) of COVID-19 cases associated with hospital admissions, and 98.9\% (88/89; 95\% Cl 93.9-100) of COVID-19 cases associated with critical care admissions occurred in women who were unvaccinated at the time of COVID19 diagnosis (Figure 1). As of the latest data release of September 30, 2021, there had been no maternal deaths associated with COVID-19 in pregnancy in Scotland (i.e. no women with confirmed COVID-19 in pregnancy who subsequently died during pregnancy or in the six weeks following pregnancy). 
By mid-October 2021, a total of 567 babies born within 28 days of maternal COVID-19 diagnosis. Of these, eight were stillbirths (deaths in utero $\geq 24$ weeks gestation) and one was a neonatal death (death within 28 days of birth) giving an extended perinatal mortality rate in this population of 15.9 per 1000 births ( $95 \% \mathrm{Cl} 7.8$ to 31.0 ; background rate in 20206.3 per 1,000 total births [95\% Cl 5.7-7.1]; background rate 20195.7 per 1,000 total births [95\% $\mathrm{Cl} 5.0-6.4]$ ]). ${ }^{16}$ All perinatal deaths occurred in women who were unvaccinated at the time of COVID-19 diagnosis.

\section{Adverse events associated with vaccination}

There have been no maternal deaths associated with COVID-19 vaccination in Scotland (i.e. no women who received COVID-19 vaccination and subsequently died during pregnancy or in the six weeks following pregnancy). By mid-October 2021, a total of 3,809 babies had been born to women who had received COVID-19 vaccination during pregnancy. Six of these births were stillbirths and eight babies died within 28 days of birth, giving an extended perinatal mortality rate of 3.7 per 1000 total births [ $95 \% \mathrm{Cl} 2.0$ 6.2], which is reassuringly in line with the background extended perinatal mortality rates of 6.3 per 1,000 total births [ $95 \% \mathrm{Cl} 5.7-7.1]$ in 2020 and 5.7 per 1,000 total births [95\% Cl 5.0-6.4]) in 2019. ${ }^{16}$

\section{Conclusion}

These data provide new national evidence of low levels of vaccine uptake in pregnancy in Scotland, at a time when the Delta variant dominated and infection rates were still increasing in pregnant women. We found that complications known to be associated with COVID-19 in pregnancy (critical care admission, perinatal mortality) were far more common in women who were unvaccinated at the time of SARS-CoV-2 diagnosis than in vaccinated pregnant women. Although COVID-19 rates were similar across all trimesters of pregnancy, and mirrored those in the general female population of reproductive age, associated hospital admissions and critical care admissions were higher in pregnant women than in the general female population of reproductive age, and highest in the third trimester. Low vaccine uptake rates in Scotland are likely to be representative of those in the U.K. and other high-income countries. ${ }^{17,18}$ Addressing low vaccine uptake rates in pregnant women is imperative to protect the health of women and babies.

\section{Declarations}

\section{Contributorship}

SJS, RW, CR and AS conceived the study. SJS, EV, CRS, UA, CM, JD, LR, CR, AS, LH, LEMH, AG, JC, M, JP, CC and RW designed the study. SJS, EV, CRS, UA, CM, LH, JD, LR, CR, AS, RM, SVK and RW drafted the protocol. JD, AG, CD, LH, LEMH, TM and JC performed data analysis. SJS, EV, CRS, UA, CM, LH, LR, CR, $A S, A G, C D, L E M H, J C, B A, C C, J P, J M$ and RW interpreted the data and revised the manuscript for important intellectual content. SJS, DM, EV, CRS, UA, CM, LH, JD, LR, CR, AS, AG, CD, LEMH, JC, BA, SVK, CC, JP, JM and RW gave final approval of the version to be published. RW acts as guarantor for the study. 


\section{Funding}

COPS is a sub-study of EAVE II, which is funded by the Medical Research Council (MC_PC_19075) with the support of BREATHE - The Health Data Research Hub for Respiratory Health [MC_PC_19004], which is funded through the UK Research and Innovation Industrial Strategy Challenge Fund and delivered through Health Data Research UK. This research is part of the Data and Connectivity National Core Study, led by Health Data Research UK in partnership with the Office for National Statistics and funded by UK Research and Innovation (grant ref MC_PC_20058) Additional support has been provided through Public Health Scotland and Scottish Government DG Health and Social Care. COPS has received additional funding from Tommy's charity and support from Sands charity. SJS is funded by a Wellcome Trust Clinical Career Development Fellowship (209560/Z/17/Z). SVK acknowledges funding from a NRS Senior Clinical Fellowship (SCAF/15/02), the Medical Research Council (MC_UU_00022/2) and the Scottish Government Chief Scientist Office (SPHSU17). BA was supported by the European Union's Horizon 2020 research and innovation programme under the Marie Skłodowska-Curie grant agreement No.813546, the Baily Thomas Charitable Fund, the Data Driven Innovation and the UK Economic and Social Research Council (ES/N018877/1) during the course of this work.

The funders had no role in the writing of the manuscript or the decision to submit it for publication.

\section{Conflicts of interests}

AS and CR are members of the Scottish Government's COVID-19 Advisory Group. AS and CR are members of the New and Emerging Respiratory Virus Threats Advisory Group (NERVTAG) risk stratification subgroup. CR is a member of the Scientific Pandemic Influenza Group on Modelling. AS is a member of the Scottish Government's Standing Committee on Pandemics and AstraZeneca's Thrombotic Thrombocytopenic Advisory Group. SVK was co-chair of the Scottish Government's Expert Reference Group on Ethnicity and COVID-19. All roles are unremunerated. RW and CR are employed by Public Health Scotland.

\section{Acknowledgments}

Our thanks to the EAVE II Patient Advisory Group and Sands charity for their support.

\section{References}


1. Zambrano LD, Ellington S, Strid P, et al. Update: Characteristics of Symptomatic Women of Reproductive Age with Laboratory-Confirmed SARS-CoV-2 Infection by Pregnancy Status - United States, January 22-October 3, 2020. MMWR Morb Mortal Wkly Rep 2020; 69(44): 1641-7.

2. Villar J, Ariff S, Gunier RB, et al. Maternal and Neonatal Morbidity and Mortality Among Pregnant Women With and Without COVID-19 Infection: The INTERCOVID Multinational Cohort Study. JAMA Pediatr 2021; 175(8): 817-26.

3. Papageorghiou AT, Deruelle P, Gunier RB, et al. Preeclampsia and COVID-19: results from the INTERCOVID prospective longitudinal study. Am J Obstet Gynecol 2021; 225(3): 289 e1-e17.

4. Wei SQ, Bilodeau-Bertrand M, Liu S, Auger N. The impact of COVID-19 on pregnancy outcomes: a systematic review and meta-analysis. CMAJ 2021; 193(16): E540-E8.

5. Knight M, Bunch K, Vousden N, et al. Characteristics and outcomes of pregnant women admitted to hospital with confirmed SARS-CoV-2 infection in UK: national population based cohort study. BMJ 2020; 369: m2107.

6. Vousden N, Bunch K, Morris E, et al. The incidence, characteristics and outcomes of pregnant women hospitalized with symptomatic and asymptomatic SARS-CoV-2 infection in the UK from March to September 2020: A national cohort study using the UK Obstetric Surveillance System (UKOSS). PLoS One 2021; 16(5): e0251123.

7. Mullins E, Hudak ML, Banerjee J, et al. Pregnancy and neonatal outcomes of COVID-19: coreporting of common outcomes from PAN-COVID and AAP-SONPM registries. Ultrasound Obstet Gynecol 2021; 57(4): 573-81.

8. Allotey J, Stallings E, Bonet M, et al. Clinical manifestations, risk factors, and maternal and perinatal outcomes of coronavirus disease 2019 in pregnancy: living systematic review and meta-analysis. BMJ 2020; 370: m3320.

9. Shimabukuro TT, Kim SY, Myers TR, et al. Preliminary Findings of mRNA Covid-19 Vaccine Safety in Pregnant Persons. N Engl J Med 2021; 384(24): 2273-82.

10. Razzaghi H, Meghani M, Pingali C, et al. COVID-19 Vaccination Coverage Among Pregnant Women During Pregnancy - Eight Integrated Health Care Organizations, United States, December 14, 2020 May 8, 2021. MMWR Morb Mortal Wkly Rep 2021; 70(24): 895-9.

11. Stock SJ, McAllister D, Vasileiou E, et al. COVID-19 in Pregnancy in Scotland (COPS): protocol for an observational study using linked Scottish national data. BMJ Open 2020; 10(11): e042813.

12. Stock SJ, Carruthers J, Denny C, et al. Cohort profile: The Covid-19 in pregnancy in Scotland (COPS) dynamic cohort of pregnant women to assess effects of viral and vaccine exposures on pregnancy. International Journal of Epidemiology 2021; In press

13. Simpson CR, Robertson C, Vasileiou E, et al. Early Pandemic Evaluation and Enhanced Surveillance of COVID-19 (EAVE II): protocol for an observational study using linked Scottish national data. BMJ Open 2020; 10(6): e039097.

14. Mulholland RH, Vasileiou E, Simpson $\mathrm{CR}$, et al. Cohort profile: early pandemic evaluation and enhanced surveillance of COVID-19 (EAVE II) database. International Journal of Epidemiology 2021. 
15. Public Health Scotland COVID-19 Daily Dashboard. Available from: https://public.tableau.com/app/profile/phs.covid.19/viz/COVID19DailyDashboard_15960160643010/Overview/viz/COVID19DailyDashboard_15960160643010/Overview. Accessed Nov 32021.

16. National Records of Scotland. Stillbirths and Infant Deaths. Available from: https://www.nrscotland.gov.uk/statistics-and-data/statistics/statistics-by-theme/vitalevents/general-publications/vital-events-reference-tables/2020/list-of-data-tables\#section4. Accessed Nov 32021.

17. UK Government Covid-19-vaccine-surveillance-report. 2021. Available from: https://www.gov.uk/government/publications/covid-19-vaccine-surveillance-report. Accessed Nov 3 2021.

18. Personal Communication Public Heath Wales.

\section{Figures}




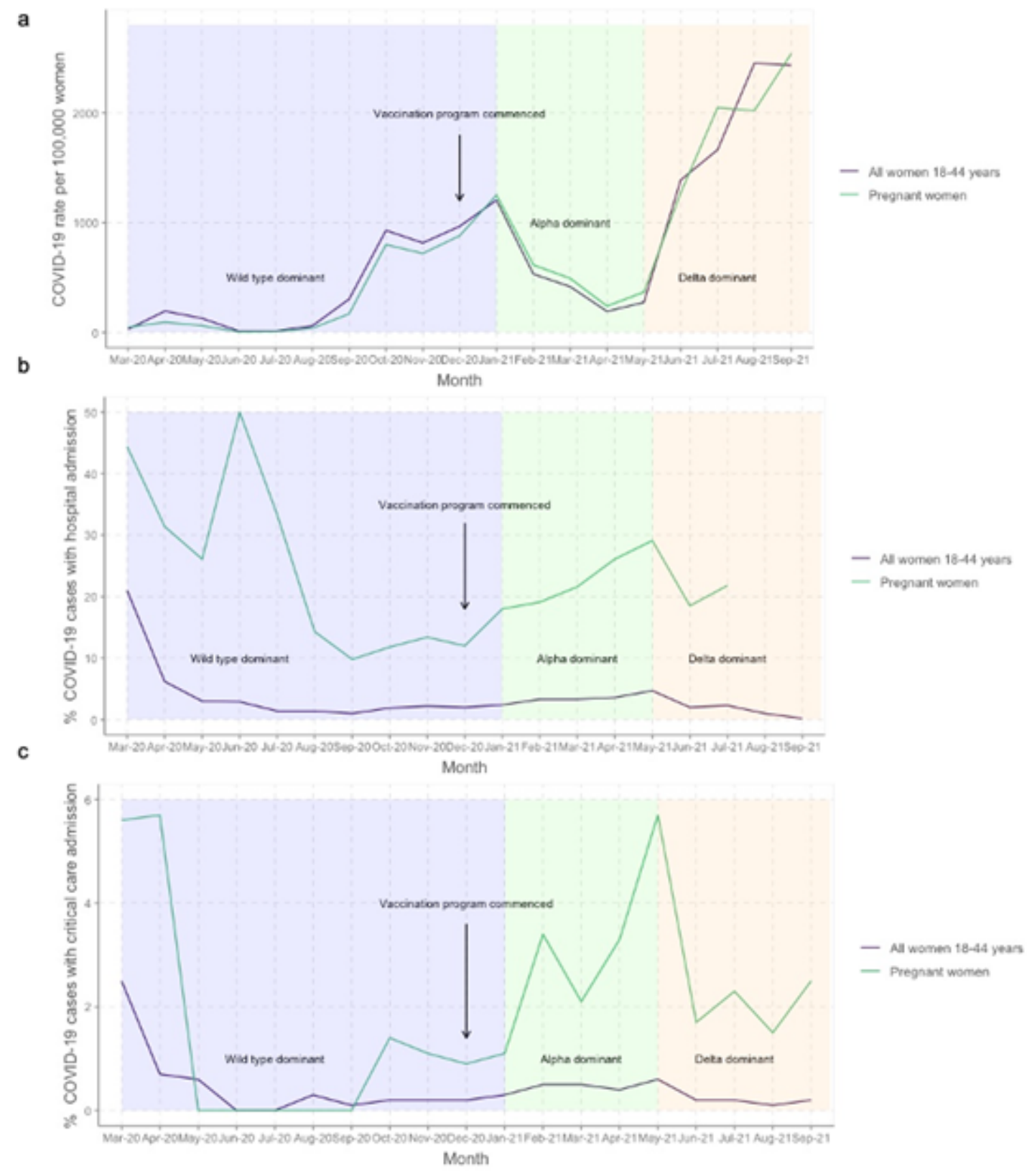

\section{Figure 1}

a. SARS-CoV-2 in pregnant women and the general population of women since March 2020 (month of the first case in Scotland) per 100,000 women. COVID-19 rates in pregnancy have a similar pattern to infection rates in the general female population of reproductive age, with variation over time reflecting sequential waves of infection and more complete case ascertainment as access to testing increased. Low rates of confirmed cases of COVID-19 in pregnant women were seen in the first wave of infection in March to May 2020, when wild type Sars-2-Cov predominated. Subsequent peaks in case numbers have been seen in October 2020 and January 2021 (alpha variant dominant) with rates increasing again in summer 2021 (delta variant dominant). b. Percentage of cases of SARS-CoV-2 with associated hospital admission in pregnant women and the general population of women since March 2020 (month of the first case in Scotland) per 100,000 women. An episode of COVID-19 was been defined as associated with a hospital admission if the woman was admitted to hospital $\leq 14$ days after the date of onset of COVID- 
19 , or if the date of onset occurred at any point during a hospital admission. Rates of COVID-19 associated hospital admissions have been consistently higher than rates in the general female population of reproductive age. General hospital admissions is incomplete for August and September due to source data latency and so these have not been included in the figure. There were very low case numbers of confirmed SARS-CoV-2 in June 2020, which may explain the very high admission rate at this point in time c. Percentage of cases of COVID-19 associated critical care admission within 21 days of diagnosis in pregnant women and the general population of women since March 2020 (month of the first case in Scotland) per 100,000 women. An episode of COVID-19 was defined as associated with a critical care admission if the woman was admitted to critical care $\leq 21$ days after the date of onset of COVID-19, or if the date of onset occurred at any point during a critical care admission. Rates of COVID-19 associated critical care admissions have been higher than rates in the general female population of reproductive age since October 2020 . The percentage of cases with associated admission was high early in the pandemic due to restricted testing (mainly people ill enough to require admission and healthcare workers before May 2020). 


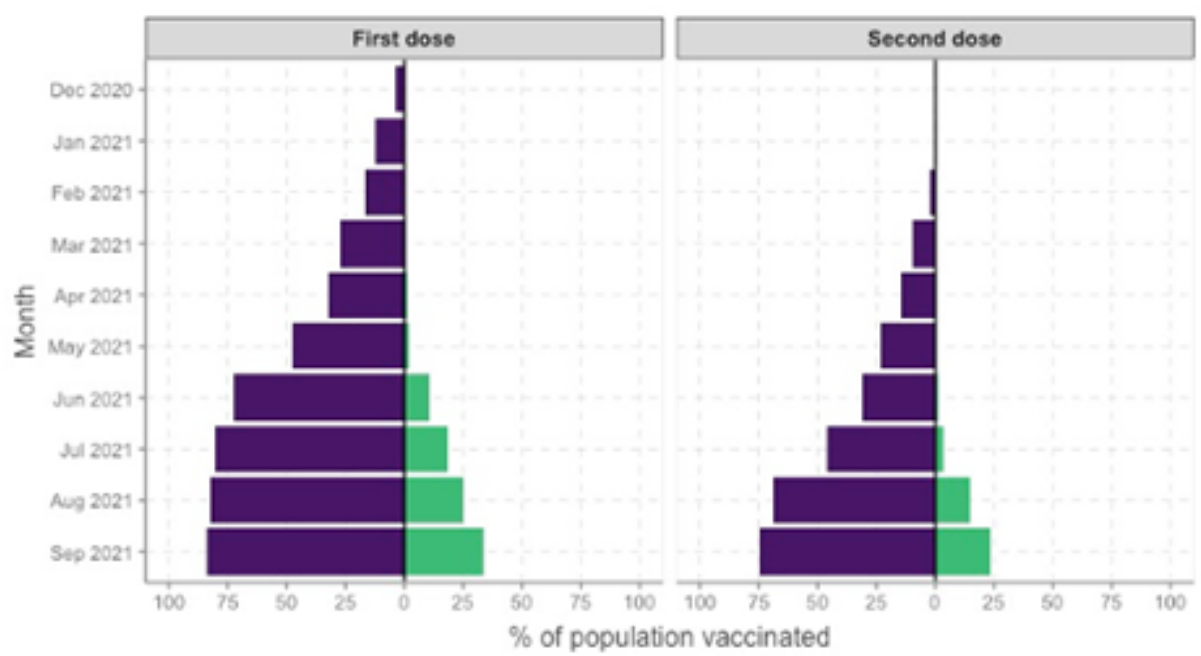

All women $18-44$ years

Pregnant women at time of birth

b

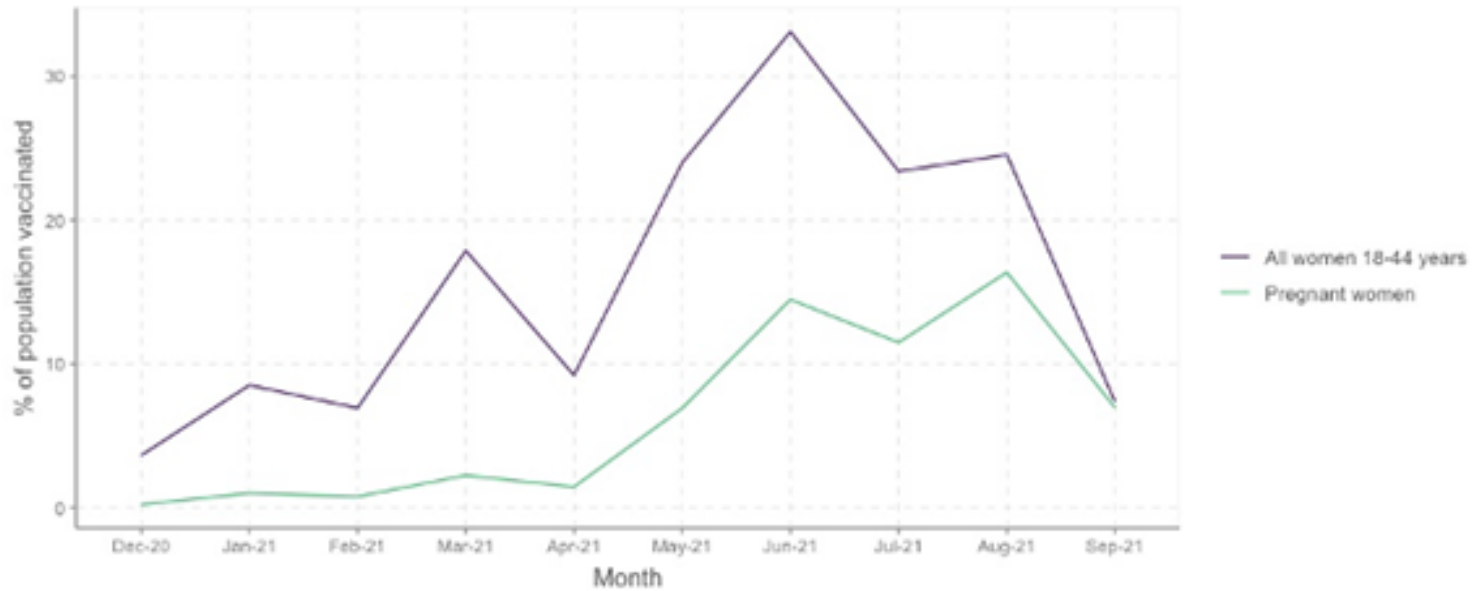

$\%$ COVID- 19 cases

c
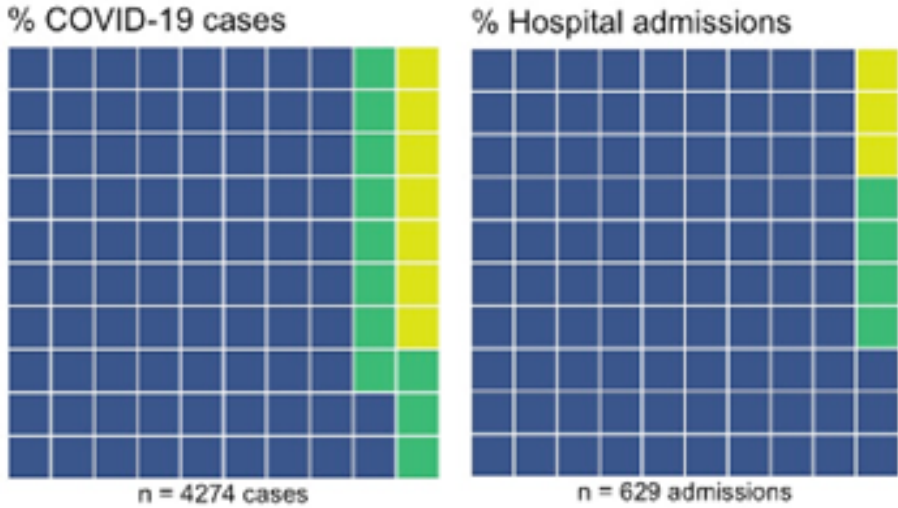

\% Critical care admissions

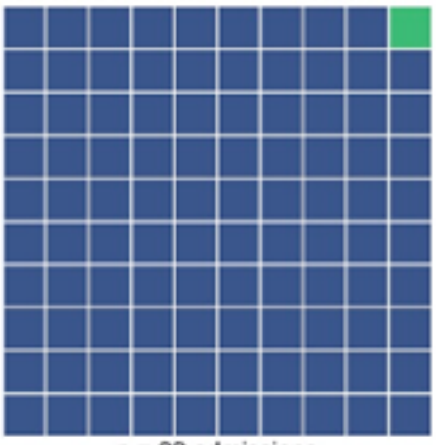

Unsosingen

Petiesy wexcrated

fuy roscinus:

\section{Figure 2}

a. Vaccine coverage in general female population in women age 18-44 years and percentage of pregnant women vaccinated before the time of birth (calculated as the number of women giving birth in the month who had received vaccination prior to or during pregnancy, divided by the number of women giving birth in that month*100). b. Monthly uptake of COVID-19 vaccination in all women 18-44 years in Scotland and in pregnant women (calculated as the number of women receiving any COVID-19 vaccination during pregnancy in the month, divided by the number of women with an ongoing pregnancy at the start of that month*100). c. Percentage of cases of COVID-19 (confirmed by SARS-2-CoV RT-PCR) in pregnancy occurring Dec 2020 to September 2021 inclusive, cases with associated hospital admission, and cases 
with associated critical care admission, by vaccination status at the date of first positive RT-PCR test. Unvaccinated is defined as no prior COVID-19 vaccination prior to the date of onset of COVID-19, or with one dose of vaccination $\leq 21$ days prior to the date of onset. Partially vaccinated is defined as one dose of vaccination $>21$ days prior to the date of onset of COVID-19, or two doses of vaccination with the second dose $\leq 14$ days prior to the date of onset. Fully vaccinated is defined as two doses of vaccination with the second dose $>14$ days prior to the date of onset of COVID-19.

\section{Supplementary Files}

This is a list of supplementary files associated with this preprint. Click to download.

- SupplementaryFile.docx 